\title{
Penetración del léxico químico en el DRAE: la edición de $1817^{1}$
}

\author{
Cecilio Garriga Escribano \\ Universitat Rovira i Virgili (Tarragona)
}

\section{INTRODUCCIÓN}

La Guerra de Sucesión marca el inicio del siglo XVIII español, con la subida al trono de los Borbones y el cambio de rumbo respecto a la política de los monarcas de la Casa de Austria. A partir de aquí, a España empiezan a llegar los nuevos aires de la llustración. Pero lo hacen con cierto retraso. En Europa, el impulso dado a las ciencias experimentales por las monarquías de la época originaba la creación de academias, gabinetes, observatorios, jardines botánicos, laboratorios, etc. Se producía así el avance y la formalización de ciencias tan importantes como la física, la mecánica, la óptica, la botánica o la química.

Para recuperar el terreno perdido, la monarquía española decide poner las bases para el desarrollo de la ciencia en la Península. Fomenta la creación de instituciones científicas, ya sean Academias o Sociedades de Amigos del País ${ }^{2}$ y potencia tanto la salida de pensionados y becarios para estudiar en los principales centros europeos, como la venida de importantes maestros extranjeros para enseñar en los centros españoles.

De esta manera, España se va incorporando al avance técnico y científico de la llustración. Y este proceso tiene unas claras repercusiones en la lengua, ya que el español, que según F. Lázaro CarReter adolecía desde el Renacimiento de la falta de léxico especializado para las ciencias y artes, adapta las diferentes terminologías, con la penetración de gran parte de las

1 Este trabajo ha sido posible gracias a una subvención de la DGICYT al Proyecto de Investigación Vocabulario de la química española en el siglo XIX (PB94-0918), dirigido por el profesor Juan Gutiérrez Cuadrado, de la Universidad de Barcelona. Una primera versión del mismo se presentó como comunicación en el XXV Simposio de la Sociedad Española de Lingüística, celebrado en la Universidad de Zaragoza.

2 El fenómeno de la aparición de estas instituciones es analizado por J. CLÉMENT $(1993,30$ y ss.). 
voces técnicas actuales ${ }^{3}$. Esto se produce fundamentalmente a través de las traducciones de los más importantes tratados científicos de la época ${ }^{4}$. De entre las ciencias que alcanzan su madurez en el siglo XVIII, ocupa un lugar destacado la química, ya que constituye un ejemplo de ciencia experimental formalizada a partir de la institución de una terminología.

Paralelamente a este proceso, la Real Academia Española de la Lengua, la primera academia creada bajo el reinado de Felipe $V(1713)^{5}$, iba trazando un camino iniciado con el Diccionario de Autoridades, que la consolidaba como el pilar fundamental de la lexicografía española. Uno de sus objetivos era fijar la lengua, las palabras, dar fe del buen uso'.

Mi propósito aquí es estudiar la vía de penetración de algunos de los términos químicos más significativos en la consolidación de esta ciencia, y su fijación en el Diccionario académico, como notario del uso. También me detendré para constatar la transformación que la nueva concepción de la química introduce en la definición de determinados elementos. Todo ello nos conducirá a un conocimiento más objetivo sobre la presencia de tecnicismos en las primeras ediciones del DRAE, $y$ al convencimiento, con K. BALDINGER (1985), de que las relaciones entre lengua y cultura son un aspecto aún no suficientemente aprovechado para los estudios lingüísti$\cos ^{7}$.

3 La razón aducida por F. LÁzARo Carreter $(1985,284)$ es que: "Por la época en que Galileo, Copérnico y Kepler dotaban al mundo de leyes naturales y matemáticas, España producía sus más asombrosas obras literarias, quedando al margen de aquellas inquietudes". Sin embargo, J. M. LóPEZ PIÑERO $(1979,15$ y ss.) explica que esto no es exactamente así, e incluye este tipo de afirmaciones en la «polémica de la ciencia española» que responde más a planteamientos ideológicos que rigurosamente históricos, y que confunde la falta de información con el vacío histórico.

4 J. CLÉMENT $(1993,42)$ constata que mientras que hasta 1750 se publican solo 38 obras en español, cinco de ellas traducciones, en la segunda mitad del siglo XVIII aparecen 82 traducciones y otros 89 originales españoles.

5 Creada y aprobada en 1713 , pero Real en 1714.

6 Felipe $V$, en su Autorización (RAE, 1726, XXI), afirma que gracias al Diccionario "[...] se conocerá con evidéncia, que la léngua Castellana es una de las mejores que oy están en uso, y capáz de tratarse, y aprenderse en ella todas las Artes y Ciencias, como de traducir con igual propriedád y valentía qualesquiera origináles". Según Álvarez de Miranda $(1992,6)$, las raíces de la Academia hay que buscarlas ya en los últimos años del reinado de Carlos $॥$.

7 K. BALDINGER $(1985,253)$ propone la integración rigurosa y sistemática de los factores extralingüísticos en la explicación de los fenómenos lingüísticos, y defiende su aprovechamiento en todo lo que corresponde a la lingüística histórica. 


\section{LA CIENCIA QUÍMICA}

La alquimia venía trabajando desde la Edad Media con los cuatro elementos aristotélicos, el agua, la tierra, el aire y el fuego. Paracelso convierte la alquimia en farmacia, como apunta J. ESTEVA DE SAGRERA (1991, 39), y así se instituye la yatroquímica, aunque en España la Contrarreforma impide el desarrollo del paracelsismo, y la medicina se mantiene fiel al galenismo tradicional ${ }^{8}$. Solo en los últimos veinte años del siglo XVII un grupo de científicos, llamados despectivamente novatores, denuncian la situación de la ciencia en España e inician un complicado movimiento de renovación?.

En Europa, la química había ido evolucionando, en busca de una explicación para el fenómeno de la combustión, que STAHL había establecido a partir de la existencia del flogisto, un principio común a todos los cuerpos combustibles. Así se desarrolló una interesante teoría que permitía explicar algunos fenómenos hasta ese momento inexplicables. Como dice $\mathrm{M}^{\mathrm{a}}$. C. CALLEJA $^{10}$, este hecho proporcionó a la química un cuerpo de doctrina del que carecía hasta entonces. En este contexto, LAVOISIER propone una nueva teoría, que niega la existencia del flogisto, y que se basa en la descomposición del aire atmosférico, que hasta ese momento se consideraba un elemento simple. Este es el punto de inflexión de la ciencia química moderna ${ }^{11}$.

En esta situación, y gracias al ambiente creado por los borbones para el desarrollo de las ciencias, de cuya utilidad eran los máximos beneficiarios ${ }^{12}$,

8 Sobre el paracelsismo aplicado a la medicina, vid. LAín ENTRALGo (1978, 289 y ss.), y (1978, 331 y ss.) sobre la yatroquímica. Vid. también J. M. LóPEZ PIÑERO (1979, 339 y ss.).

9 El movimiento renovador de finales del siglo XVII es examinado en especial por J. M. LóPEZ PIÑERo (1979, 387 y ss.).

10 Vid. Ma . C. Calleja (1992), 7.

11 Vid., para este momento, B. WoJTKOWIAK (1987), y para la importancia de LAVOISIER, M. GOUPIL (1992). En B. BeNSAUDE-VINCENT (1995) se puede encontrar un análisis del cambio de paradigma científico que se produce en la química, no como un proceso acumulativo de conversiones individuales a partir de Lavoisier, sino como una empresa, colectiva e internacional en la que influyen aspectos políticos, religiosos, culturales o nacionalistas. En cualquier caso, deja claro que la adopción de la nueva nomenclatura no necesariamente conlleva un consenso doctrinal.

12 D. García Fernández, traductor de los Elementos del arte de teñir de BerthoLLET, dice en el "Prefacio" (1795, VIII): "[...] esta es la causa de haber mandado traducir la presente obra del ilustre BERTHOLLET, á fin de que la Nacion se aproveche de las luces y descubrimientos de sus mismos émulos para el fomento de nuestra industria". Se trata de un significativo texto en el que se dice que habrá mayor ventaja en aprovechar las enseñanzas de este método que en conquistar una provincia, y que esta es la manera de "vivificar la industria, que tanto tiempo ha yace entorpecida por la falta de principios que reyna en nuestras fábricas" (1795, IX). Por cierto, esta traducción se imprime en la Imprenta Real. 
empiezan a aparecer en España los primeros laboratorios químicos, como la Cátedra Química de Vergara (1779) ${ }^{13}$, la de Madrid (1788), la de Valencia (1791), la de Segovia (1792) y la de Cádiz (1795). Las instituciones militares son otra vía para el desarrollo de la ciencia. Sirva como ejemplo el laboratorio del Real Colegio de Artillería de Segovia ${ }^{14}$. Y para regentar estos laboratorios se trae a químicos extranjeros de la talla de Luis Proust o Francisco Chavaneau, quienes formarán a los nuevos químicos españoles, explicando las teorías de LAVOISIER ${ }^{15}$. Como apunta R. GAGO (1982, XLVII), esta moderna visión de la química no encontrará resistencia en España debido a que prácticamente no había seguidores de la teoría del flogisto, como podía ocurrir en Europa ${ }^{16}$.

Pero para la enseñanza, claro está, hacían falta los textos que explicaban las nuevas teorías. $Y$ estos no se hicieron esperar. Solo un año después de su publicación en francés, aparece en español la traducción de GUTIÉRREZ BUENo, en 1788, del Método de la nueva nomenclatura química, de G. MORVEAU, A. L. LAVOISIER, C. L. Berthollet y A. FourCRoy, manifiesto fundamental de la nueva propuesta ${ }^{17}$. El Tratado elemental de química de LAVOISIER, en traducción de J. M. MuNÁRRIZ, no se publica en español hasta 1798, nueve años después de que apareciera en francés. Pero entre estas dos obras, en solo diez

13 Para la historia de la cátedra química de Vergara, vid. I. Pellón y R. GaGo (1994).

14 J. CLÉMENT $(1993,29)$ señala la consideración de gran parte de los avances científicos como secretos de Estado, útiles a la defensa, como razón de lo que llama militarización de la ciencia. Por otro lado, la autoridad tenía más facilidad para imponer una reforma vigilida en las aulas militares que en las universitarias, más conservadoras y menos intervenidas por la Monarquía. Alguna referencia más a las academias militares en S. Riera $(1992,12)$. M. Peset y J. L. Peset $(1992,20)$ muestran cómo las ciencias se enseñaban mejor en las academias militares o en los reales colegios de cirugía que en la universidad. Concretamente sobre el Real Colegio de Artillería de Segovia, vid. M. D. HERRERO (1993).

15 Sobre L. Proust y sus actividades en Segovia, vid. R. GaGo (1990). Para un estudio léxico de sus Anales, vid. C. GarRICA (1996b).

16 No todos los pensadores estaban seguros de que la mayoría de los países consintieran adoptar la nueva nomenclatura, no porque no fuera apropiada, sino porque las razones políticas constituían un obstáculo en la situación histórica del momento. Así lo demuestran J. Gutiérrez y J. L. Peset (en prensa) con palabras de J. A. Alzate (1831): " ¿Cómo quiere V. que las naciones concurran a establecer un idioma químico? Basta que sea invención francesa para que los ingleses la detesten, y continúen en seguir el establecido idioma químico". Todo ello en fechas en que muchos de estos términos ya estaban fijados en el DRAE.

17 Esta traducción, como señala J. M. López PIÑERO $(1982,49)$, es simultánea a la inglesa y anterior a la de los demás países. Para M. J. Calleja $(1992,14)$, GutiérRez BUENO debe ser considerado como el introductor de las teorías de LAVOISIER en España. Después hubo otras traducciones, vid. C. GaRRIGA (1997b) donde se realiza un estudio comparativo. 
años, se publican en español los tratados más importantes de la química moderna ${ }^{18}$, lo que hace incorporarse a la ciencia española, y con ella al español, a las más avanzadas corrientes europeas. Téngase en cuenta que la nueva nomenclatura química es, sobre todo, una terminología, como constataba el mismo LAVOISIER $(1788,5)$ al decir, según la traducción de GUTIÉRREZ BUENO, que "[...] un método analítico es una lengua; una lengua es un método analítico, y estas expresiones son en cierto modo sinónimas", y nuestros traductores eran plenamente conscientes de ello ${ }^{19}$. Todo esto en un ambiente propicio y favorable a las nuevas teorías, que hacía prever, como apuntan E. PORTELA y A. SOler $(1992,96)$, un desarrollo de la química similar al de los demás países, si no hubiera sido por las negativas condiciones que se dieron en el primer decenio del S. $X I^{20}$.

18 No en vano J. CLÉMENT $(1993,42)$ habla del Siglo de las Luces como del siglo de las traducciones. Algunas de las más significativas para la química son los Elementos de química teórica y práctica de G. Morveau, MareT y DuRANDE (trad. Melchor DE GUARDIA Y ARDÉVOL, 1788); las Lecciones de química teórica y práctica de G. MORVEAU, MAREt y DURANDE (trad. Tadeo LOPE y ACUILAR, 1789); los Elementos de química de J. A. ChAPTAL (trad. Hyginio Antonio LoRente, 1793-94), los Elementos de Historia Natural y de Química de M. FourCroy (trad. Tadeo LoPe AcuILAR, 1793-95); los Elementos del arte de teñir de A. BERTHOLLET, con una nueva traducción del Método de la nueva nomenclatura química (trad. Domingo GARCíA FERNÁNDEZ, 1795); y el Arte de fabricar el salino y la potasa de A. L. LaVoISIER (trad. Juan Manuel MunÁrRIZ, 1795).

19 Sirva el ejemplo de GutiérRez Bueno que, en la Advertencia al Método de la nueva nomenclatura química de MORVEAU, LAVOISIER, BERTHOLLET Y FOURCROY (1788, II-IV) escribía: "A primera vista se presenta, que á cada voz nueva se debe haber buscado en nuestro castellano otra igualmente significativa y propia, que esté autorizada por los mejores Diccionarios de la Lengua, y por los Autores más célebres. Mas á poca reflexîon, se conocerá la imposibilidad de esta empresa, pues no hay quien ignore la escaséz de voces que padece nuestra lengua en punto de Ciencias Naturales y Artes. Fuera de que, aunque á costa de sumo trabajo, se hubieran querido acomodar aquellas voces que menos disonasen á un oido español, se hubiera hecho una obra enteramente contraria al intento de los autores de esta nomenclatura, y absolutamente inutil para el objeto que se propusieron en inventarla. A la verdad, su animo en crear este modo de nombrar las substancias químicas, no fué para añadir estas voces á su idioma nativo, sino para mejorar y reformar el lenguage de la química, y hacerle por este medio comun á todos los Paises, y facilitar la comunicacion de los trabajos de los Profesores y Aficionados a ésta utilisima ciencia".

20 Baste citar los nombres de A. de Martí I Franquès o de J. M. de Aréjula no solo como seguidores de LAVOISIER, sino como críticos de su teoría desde la propia teoría, con aportaciones originales a la química moderna. Sobre Aréjula Vid. R. GACO, J. L. Carrillo y L. García Ballester (1974). Martí i Franquès es tratado por A. Quintana (1935); para más datos sobre su figura, vid. las referencias de M. D. EsQué y A. M. CARMONA (1995) y A. NieTo (1996). Para los motivos que condujeron al «período catástrofe» de la actividad científica española, entre 1808 y 1833, vid. J. M. LópEZ PIÑERO (1992, 14-15). 


\section{LA LEXICOGRAFÍA}

Por su lado, la Real Academia Española de la Lengua se había planteado como primer objetivo la realización de un diccionario, tal como lo expresa en sus Estatutos ${ }^{21}$. Y así, entre 1726 y 1739 se publican los seis tomos del Diccionario de Autoridades, que van a dotar al español de un instrumento del que ya disfrutaban otras lenguas de cultura, como el italiano y el francés. Según la RAE, el Diccionario recoge todas las voces de la lengua "con algunas pertenecientes à las Artes y Ciéncias" (AUTORIDADES, II). Y aunque J. CLÉMENT señala que este primer Diccionario incluye bastantes tecnicismos de la época $(1993,49)$, la Academia tenía el propósito de recopilar un diccionario de voces de carácter científico y técnico, como lo anuncia en su Prólogo:

De las voces próprias pertenecientes à Artes liberales y mechánicas ha discurrido la Académia hacer un Diccionario separado, quando este se haya concluído; por cuya razón se ponen solo las que han parecido mas comúnes y precisas al uso, y que se podían echar menos (AUTORIDADES, $V$ ).

La idea se mantiene mientras dura la elaboración de este primer diccionario, pero lo cierto es que la Corporación, una vez acabado AUTORIDADES, se dedicó a otros menesteres como la ortografía o la gramática ${ }^{22}$. Fue E. TERREROS (1786) el que elaboró un diccionario "con las voces de ciencias y artes" -así se titulaba-, cuya influencia sería enorme en todo el siglo XIX. Sus tres tomos, más el cuarto de correspondencias, incluyen, según M. ALvAR EZQUERRA, unas 60.000 entradas, frente a las 42.500 de AUTORIDADES ${ }^{23}$. Pero el de TERREROS no es solo un diccionario de ciencias y artes, sino general de la lengua castellana, en el que, eso sí, se presta una atención especial a este vocabulario; por eso:

21 El 24 de enero de 1715 la Academia aprobaba sus Estatutos, en cuyo capítulo primero se afirma que la Academia $(1726$, XXII) "[...] tiene por conveniente dár princípio desde luego por la formación de un Diccionario de la léngua, el mas copioso que pudiere hacerse". Sobre la fundación de la Academia y sus objetivos, vid. D. Fries (1989), y sobre las vicisitudes por las que pasó la elaboración de AUTORIDADES, vid. F. LÁZARO CARRETER (1980).

22 Aún en la Continuación de la Historia de la Real Academia Española que figura como prólogo al tomo sexto de AUTORIDADEs (1739), se anuncia que: "Prosigue la formación del Suplemento, y corrección de estos seis Tomos, à que se seguirá otro Diccionário de Artes, y Ciencias, en que à demás de notarse las voces pertenecientes à cada una, se añadirá una breve Explicación, que puede dar noticia de sus principales elementos à todos los Españoles, que por carecer de otros Idiomas, no puedan adquirirla en ellos [...]".

23 Vid. M. Alvar EzQuerRa (1987), IX. Como este autor constata (x-xI) Terreros recoge prácticamente todas las voces del inventario académico, hacia el que tiene en todo momento una actitud elogiosa. 
[...] se hallarán en esta obra multitud de voces, y que se podrán acaso contar por millares, unas que parecerán extrañas buscadas en las artes, y que jamas se dieron á luz, nunca vieron otra, ni lograron mas extension que la corta que le daba el taller del sastre, del carpintero, del tejedor, del guarnicionero, del tornero y de otros menestrales sin número, cuyas son y cuyo exâmen he hecho comunmente por mí mismo, deseando así con mas seguridad y certidumbre comunicar al público un tesoro, que tenia enmedio de sí sin saberlo ni conocerlo [...] $(1786$, xi).

Y es que Terreros, aunque también incluye citas de los clásicos para autorizar las voces, es consciente de la imposibilidad de documentar literariamente los términos técnicos, sobre todo por la velocidad con que progresa la ciencia ${ }^{24}$. Su impulso lleva hacia el diccionario enciclopédico, y abre un debate que perdurará en la lexicografía de todo el siglo $X I X^{25}$.

La Academia, por su parte, había decidido publicar su Diccionario en un tomo (DRAE-1780), eliminando abreviaturas y citas de autores, ante la lentitud con que se desarrollaba la revisión de AUTORIDADES, del que ya había editado un volumen (1770) ${ }^{26}$. A partir de ahí, el Diccionario en un tomo se va reeditando, con la incorporación de las revisiones que se iban haciendo para la segunda edición de AUTORIDADES. Así aparecen las ediciones del DRAE de 1783, 1791 y 1803, y habrá que esperar catorce años para encontrar una nueva edición, la de $1817^{27}$. Mientras, la Guerra de la Independencia, la vuel-

24 Dice TerReros (1786, XIV) en su prólogo: "Es cosa tambien muy cierta que hay multitud de máquinas, invenciones y noticias, con que la curiosidad y luces de nuestro siglo y los inmediatos nos han enriquecido, que no conocieron los pasados, de modo que apénas hai arte ni ciencia que no haya adelantado en su esfera y ganado mucho terreno, al mismo tiempo que es sin controversia que á cada invencion, á cada máquina y pieza de ellas es menester acomodarle algun nombre".

25 Este debate se refleja aún cien años después, en el Congreso Literario Hispano-Americano de 1892, como explican J. A. Pascual y J. Gutiérrez Cuadrado (1992). Más detalles sobre las características del diccionario de TerReros en la "Presentación" de M. Alvar Ezquerra (1987) a la edición facsímil.

26 Estos razonamientos se hallan en el "Prólogo" a la primera edición (DRAE-1780). También resulta interesante la "Introducción" de M. SECO (1991) a la edición facsímil.

27 Vale la pena citar el diccionario de A. CAPMANY (1805) por ser un diccionario francés-español -la terminología química llega desde el francés-, y por incluir un Suplemento al que se destierran las voces de ciencias. Dice CapMANy $(1805, \mathrm{xv})$ que "el diccionario de la fisica, la chîmica, la anatomía, la medicina, la farmácia, la botánica, la pintura, y arquitectura, es de todas las naciones cultas", y sus voces se pueden adaptar a cualquier lengua con solo cambiar las terminaciones; de esta manera contradice a los que afirman que la lengua castellana es estéril para las materias filosóficas y científicas. Pero de los nuevos términos químicos, como se verá, solo gas se introduce en CAPMANY antes que en el DRAE-1817. También aparece azoote pero con el significado de 'mercurio'. Sobre el diccionario de CAPMANY, vid. el trabajo de C. RoIC (1995). 
ta de Fernando VII, y la práctica renuncia a seguir con la reedición de AUTORIDADES: el DRAE-1817 se convierte en la primera edición que aparece sin la indicación "reducido á un tomo para su mas fácil uso"28.

Pero estas circunstancias van acompañadas de cambios más profundos en la estructura del diccionario, por ejemplo en lo que se refiere a los tecnicismos $^{29}$. Por ejemplo, se advierte en el "Prólogo" (DRAE-1817) que:

Se han aclarado y rectificado muchas definiciones, especialmente en los articulos que pertenecen á ciencias naturales, en las que los adelantamientos de estos últimos tiempos han aclarado y corregido diferentes equivocaciones que antes eran comunes.

Y así es, al menos en lo que a la ciencia química se refiere. Es cierto que habían pasado treinta años desde que empezaron a llegar a España las nuevas propuestas, pero las circunstancias históricas y el carácter del propio Diccionario demoran hasta esta edición la introducción de los cambios ${ }^{30}$. Veremos a continuación en qué consisten.

\section{EL LÉXICO QUÍMICO EN EL DRAE}

La nueva visión de la química se materializa en el Diccionario en dos aspectos: el cambio en la concepción de los elementos tradicionalmente admitidos como simples, y la aparición de otros nuevos a los que había que darles nombre. La aplicación sistemática del método científico pone en cuestión los viejos axiomas, y demuestra empíricamente sus errores. Pero no es fácil que los nuevos descubrimientos se admitan, y así se lamenta LAVOISIER lo cito, en adelante, por la traducción de J. M. MUNÁrRIZ-:

28 Según el CONDE DE LA VIÑAZA $(1893,1508)$ "Tiráronse 6.000 ejemplares, que se agotaron en muy breve tiempo, pues el público esperaba ansioso esta edición primera, que se hizo después de la invasión y guerra francesa". El "Prólogo" de la propia edición (DRAE-1817) también da cuenta de estas vicisitudes.

29 Otros aspectos importantes, señalados en el "Prólogo" del DRAE-1817 son la simplificación de las marcas de uso, la reordenación de las expresiones pluriverbales, el perfeccionamiento de las correspondencias latinas, y la adopción de la nueva ortografía propuesta por la Academia.

30 La Academia en el "Prólogo"(DRAE-1817) expresa un amago de autocrítica al referirse al aumento de dicciones "[...] que la autoridad de nuestros mejores escritores ó el uso comun, constante y continuado de las personas cultas obliga á admitir en el Diccionario, á pesar de la pausada circunspeccion con que en esto procede la Academia, y que quizá parecerá á algunos excesiva". Para el debate que a partir de esta edición se inicia sobre la conveniencia o no de abrir el Diccionario a los tecnicismos y darle un carácter más enciclopédico, vid. M. Alvar (1992), E. ANGLADA y Ma BARGALló (1992) y los ya clásicos trabajos de M. SECo (1987) y (1987b). 
El empeño que tenemos de que todos los cuerpos naturales se compongan únicamente de tres ó quatro elementos, es una preocupacion heredada de los filósofos Griegos. La admision de quatro elementos para la formación de todos los cuerpos conocidos por sola la diversidad de sus proporciones, es una pura hipótesis imaginada mucho antes que se tuviesen las primeras nociones de la Física experimental y de la Química. Se carecía de hechos, $y$ sin ellos se formaban sistemas; $y$ ahora que los tenemos, parece que nos empeñamos en no admitirlos, si no se conforman con nuestras preocupaciones $(1798, \mathrm{x})$.

Lexicográficamente se va a poder constatar este fenómeno en el DRAE1817, en algunas de cuyas definiciones se hallarán comentarios extralexicográficos a los recientes cambios acaecidos, comentarios que marcan un cierto distanciamiento hacia las nuevas teorías. Empecemos con tres de los elementos considerados tradicionalmente como simples: el agua, el aire y el fuego. El agua era definida en AUTORIDADES COMO:

Elemento principál entre los quatro. Los mas de los Philósophos sintieron era uno de los principios de la naturaleza. Dividese en dos especies, naturál, y artificiál: la naturál es la que cae de las nubes, la del mar, la de los rios, arroyos, fuentes y pozos; y la artificiál la que es compuesta, como agua de azár, agua rosada, \&c. (AUTORIDADES, s.v. agua).

El DRAE-1803 muestra una definición mucho más descriptiva y moderna, basándose en sus cualidades y propiedades, al decir que es un 'Cuerpo fluido, húmedo, y transparente, sin olor ni color, y capaz de apagar el fuego', sin hacer referencia al concepto de sustancia simple. Y es que LAVoIsIER había escrito que:

El agua se habia tenido hasta nuestros dias por una sustancia simple; y no halláron dificultad alguna los antiguos para calificarla con el nombre de elemento; en efecto debian tenerla por tal, pues no habian llegado a descomponerla $[\ldots](1798,60)$.

En la edición del DRAE-1817 se nota por fin la influencia de las palabras de LAVOISIER, sin que por ello el lexicógrafo se comprometa con la nueva concepción química ${ }^{31}$, al definir el agua como:

Sustancia en su estado mas comun fluida, elastica, trasparente, insípida y sin olor, la mas abundante derramada por todos los cuerpos de la naturaleza. Hasta nuestros dias fue reputada por simple, y como tal por uno de los elementos ó principio de los cuerpos (Drae-1817, s.v. agua).

Lo cierto es que el agua no se define como un compuesto de oxígeno e hidrógeno hasta el DRAE-1869, en una acepción marcada como propia de la 
química, aun cuando LAVOISIER $(1798,67)$ ya la había definido como tal, y MARTí I Franquès lo había escrito en español aún antes ${ }^{32}$. El caso del término aire aún es más paradigmático, porque hasta el DRAE-1803 se definía como 'Uno de los quatro elementos. Cuerpo ligero, fluido, transparente, capaz de compresion y dilatacion ${ }^{\prime 3}$. Sin embargo en el DRAE-1817 se convierte en:

Fluido elástico y transparente que llena y constituye lo que llamamos atmósfera. Durante mucho tiempo se creyó que fuese una sustancia simple ó no compuesta de otras, y como tal se contaba por uno de los cuatro elementos; pero en el dia está ya demostrado que consta de otros tres fluidos, conocidos con los nombres de oxigeno, hydrogeno y azoe (Drae-1817, s.v. aire).

Ahora sí que la Academia se alinea con las nuevas propuestas, porque la descomposición del aire es una de las piezas claves de la química moderna, ya que a partir de ahí se aíslan otros elementos desconocidos hasta entonces, algunos de ellos de gran importancia terminológica ${ }^{34}$. El tercer concepto tradicional es el del fuego, definido por el DRAE-1803 como 'Uno de los quatro elementos que tiene por calidad esencial calentar y alumbrar'35, acepción que se mantiene en el DRAE-1817, pero que se ve aumentada con una segunda, marcada como propia de la física y de la química: 'El calórico y el fluido luminoso' (Drae-1817, s.v. fuego); el calórico es otro de los nuevos conceptos introducidos por la química de LAVOISIER, al que luego me referiré.

31 Sí que lo hace, en referencia al agua, al definir hidrógeno, como luego se verá. En M. SIMÓN y M. IZQUIERDo (1995) se estudia una memoria de Lavoisier de 1782 en la que se concibe el agua como compuesto, pero en la que aún no se produce un rechazo expreso del flogisto, quizá porque -apunta el estudio- LAVOISIER aún no estuviera del todo seguro de que no fuera necesario para explicar la relación entre el oxígeno y el agua.

32 Vid. A. Martí I Franquès (1878), 14, quien dice "El agua pues debe ser excluida de la clase de los elementos, siendo indubitable formacion por la mescla de los aires inflamable, i desflogisticado, cuias dos substancias serán sus dos principios constitutivos, como lo jusga el Sor. LavoIsier [...]". TeRreros (1786), s. v. agua, la definía como 'uno de los cuatro elementos comunes', pero eso no debe extrañar, ya que, según M. Alvar EzQUeRRa (1987, IX), TerReros empezó a trabajar en el diccionario hacia 1745, y el primer tomo se imprimió ya en 1767. Queda interrumpido hasta 1786 debido a la expulsión de los jesuitas, orden a la que TERREROs pertenecía.

33 DRAE-1803, s. v. ayre. CAPMANY (1805) aún define el aire (s. v. air) como 'uno de los quatro elementos [...]'.

34 En el DRAE-1822 la definición cambia y toma la marca de Quím. 'química', y en el DRAE-1869 se establece la proporción de oxígeno y ázoe en el aire, una proporción que había fijado MarTí I Franquès (1790) corrigiendo los datos de Lavoisier.

35 DRAE-1803, s. v. fuego. La definición había evolucionado desde AUTORIDADES, donde se definía (s. v.) como 'El mas caliente, mas bello, mas leve, y mas activo de los elementos. Es un cuerpo luminoso, sumamente cálido, y moderadamente seco, y 
A partir de aquí, se puede mencionar una serie de términos en cuya definición se aprecia la huella de la nueva concepción de la ciencia química. Se trata de casos como el acero o el arsénico, en los que se habla del oxígeno o del proceso de oxigenación, introducidos por LAVOISIER. El ácido, por ejemplo, era definido en el DRAE-1803 simplemente como 'Quím. Sal mordicante y disolvente, cuyas propiedades son contrarias á las del álcali'(DRAE-1803, s.v. ácido). En el DRAE-1817 aparece como:

Quím. Cuerpo que en virtud de la combustion ú oxigenacion ha adquirido el gusto agrio, la propiedad de enrarecer muchos colores azules vegetales, la fuerte atraccion para la mayor parte de los cuerpos, y el formar sales combinado con ciertas sustancias (s.v. ácido).

El artículo se completa con la definición de las expresiones ácido carbónico ${ }^{36}$, ácido marino, ácido muriático, ácido nítrico, ácido sulfúrico ${ }^{37}$ y ácido vitriólico. En los textos aparecen también los adjetivos derivados acidificable (Morveau, LaVoisier, Berthollet, FourCroy, 1788, 19), acidificante (A. Martí I Franquès, 1787, 19) y acidificado (Morveau, LaVoisier, Berthollet, Fourcroy (1788) 13 $)^{38}$. Téngase en cuenta que la formación de ácidos a partir del oxígeno -más adelante me referiré a ello- es una de las cuestiones importantes planteadas por la nueva química, y que el término ácido se convierte en fundamental para la nueva nomenclatura, como el mismo LAVOISIER explica:

La palabra ácido será el nombre genérico, y cada uno de ellos se distinguirá despues en la lengua, como lo está en la naturaleza, por el nombre de su base ó de su radical. Por manera que llamaremos ácidos en general a los resultados de la combustion ú oxigenacion del fósforo, del azufre y del carbon; llamando ácido fosfórico al primero, ácido sulfúrico al segundo, y ácido carbónico al tercero $(1798,49)$.

consta de una matéria sutilíssima violentamente agitada. Entra insensiblemente en la composicion de todos los cuerpos naturales, particularmente de los que son animados: dá calór à toda la naturaleza, y dél se componen los fuegos grosséros que se extrahen de los mixtos'. Ya TerReros (1786), s. v., la simplifica en 'Elemento calido, y seco, que entra en la composicion de todos los cuerpos naturales, principalmente de los animados'.

36 Se define como 'El que resulta de la combinacion del oxigeno con el carbon', en DRAE-1817, s. v. ácido.

37 Se define como 'El que resulta de la combustion ú oxigenacion del azufre. Es de un uso muy general en las artes', en DRAE-1817, s. v. ácido.

38 Solo proporciono una documentación, a modo de ejemplo, de las muchas que se pueden hallar. 
Se pueden destacar otras voces que, como las anteriores, ya aparecían en la edición del DRAE-1803, pero en las que se aprecian diferencias significativas en las definiciones que presentan en el DRAE-1817, al utilizar para explicarlas las propiedades observadas a través de la experimentación. Se trata de casos como el azufre, la cal, el éter, el fósforo, el molibdeno, la potasa o el concepto de metal. Por ejemplo, del fósforo se decía en el DRAE-1803:

Se da generalmente este nombre á todas las substancias capaces de dar luz en la obscuridad, como los gusanos de luz, la madera podrida, la piedra de Bolonia, \&c. (s.v. fósforo).

Y en el DRAE-1817 se transforma en:

Materia sumamente combustible, ligera, quebradiza y de color de caramelo, que se derrite en el agua caliente, luce en la oscuridad, se inflama muy fácilmente y despide un olor particular. Se extrae comunmente de los huesos, y tiene diversos usos (s.v. fósforo).

Sirva como ejemplo también el del éter, definido en el DRAE-1803 como 'Fís. La substancia fluida y sutil en que se supone se mueven los líquidos celestes' (s.v. éter), frente a la definición del DRAE-1817, que cambia el concepto por otro mucho más científico:

Fís. y Quím. Licor tan claro y cristalino como el agua mas pura, muy ligero, inflamable, oloroso, poco soluble en el agua y tan volátil, que arrojado al aire ó echado en la mano desaparece inmediatamente dejando fria la parte. Se emplea en diferentes experimentos y es medicinal (s.v. éter).

Junto a estos cambios, se produce la incorporación de nuevos términos, introducidos en el DRAE en esta edición, y que están en la base de la nueva concepción de la química. Sin duda el más importante es el oxígeno, obtenido a partir de la descomposición del aire, y definido en el DRAE-1817 como:

Quím. Uno de los principios ó cuerpos simples descubierto modernamente por los químicos, que sirve principalmente para la respiracion, entra en la composicion del aire atmosferico, y por eso se llama también vital y se combina con otras sustancias, y señaladamente con las que forman varios ácidos, por cuya propiedad se le impuso aquel nombre (s.v. oxígeno).

En esta definición se recogen, en efecto, las principales características que LAVOISIER había descrito para el oxígeno, como se puede ver en el siguiente párrafo del Tratado elemental de química: 
A la base de la parte respirable del ayre le hemos dado el nombre de oxî-

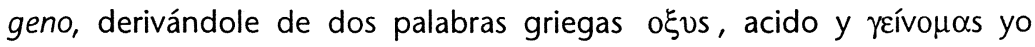
engendro; porque en efecto una de las propiedades mas generales de esta base es formar ácidos, combinándose con la mayor parte de las sustancias $(1798,38)$.

Como se puede observar, el DRAE-1817 da por bueno el razonamiento del oxígeno como 'engendrador de ácido', aun cuando otros químicos españoles, como J. M. DE ARÉjULA, habían contradicho con acierto la teoría de la acidez de LAVOISIER ${ }^{39}$. Pero el término oxígeno había sido ya aceptado por toda la comunidad científica ${ }^{40}$. Junto a oxígeno, aparecen en esta edición los derivados oxigenado y oxigenar ${ }^{41}$.

El agua, como hemos visto, ya no era un elemento simple, sino compuesto por un lado de oxígeno, y por otro de hidrógeno. El término hidrógeno sigue el mismo procedimiento para su formación que el anterior, y así lo argumenta LAVOISIER:

[...] el agua ademas de tener al oxîgeno por uno de sus principios, igualmente que otras muchas sustancias, contiene otro que le es peculiar y su radical constitutivo; y siendo preciso darle nombre, ninguno nos ha parecido mas propio que el de hydrogeno, que es lo mismo que generador de

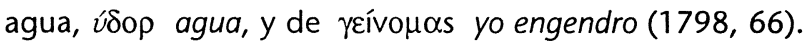

El término hidrógeno, como el de oxígeno, también aparece por primera vez en el DRAE-1817, con la definición:

Quím. Sustancia simple, que es uno de los principales constitutivos del agua, de los aceites y de otros cuerpos (s.v. hidrógeno).

Ya me he referido a la descomposición del aire como uno de los pilares de la nueva química. Estaba constituido por aire vital u oxígeno, pero también por aire no respirable, cuya principal propiedad conocida, como explicaba

39 Davy descubre en 1810 que el ácido clorhídrico estaba compuesto únicamente por cloro e hidrógeno. Este hecho acaba, como dice R. GACo (1982, LI, n. 31), con la teoría de la acidez.

40 Como alternativa se habían propuesto otros términos como arxicayo o gas comburente, que atribuían a este gas la cualidad de ser responsable de la combustión. Ninguno de ellos llega a aparecer en el DRAE, aunque se pueden encontrar en el diccionario de E. CHAO (1864), y gas comburente, además, en el de R. J. Domíncuez (1846). En cambio, oxygino es utilizado ya por MARTí I Franquès $(1787,19)$.

41 Otros derivados se van incorporando en ediciones posteriores, como ocurre con óxido (DRAE-1822), o con oxidación y oxidar (DRAE-1852). CAPMANY (1805) aún no recogía oxígeno en su diccionario francés-español. 
LAVoIsier era la de "quitar la vida a los animales que le respiran" (1798, 39), y de ahí su nombre, ázoe, "de la a privativa de los Griegos, y de $\xi \omega \eta ́$ (sic), que significa vida" $(1798,38)$. Inicialmente hay dificultades para fijar el término, ya que la forma original francesa azote creaba problemas de homonimia, y así lo expresa GutiérRez Bueno (vid. Morveau, LaVoisier, Berthollet y FOURCROY, 1788, v), primer traductor, quien se decide por azoote, aunque en alguna ocasión utiliza la forma ázoo ${ }^{42}$. En cambio, L. Proust (1795) emplea azote, sin alteración alguna respecto al francés, en el Prólogo de sus Anales. El término ázoe se puede documentar en la traducción de GARCía FERNÁNDEZ de los Elementos del arte de teñir de BERTHOLLeT (1795), en el Diccionario para la nueva Nomenclatura Chîmica que añade a esta obra ${ }^{43}$. En el DRAE-1817 aparecen las dos formas, ázoe y azote. La última remite a la primera, que se define como:

Quím. Sustancia simple que unida al calórico ó á la materia del calor se reduce a fluido aeriforme ó gas azoe, en el cual no pueden vivir los animales ni quemarse ningun cuerpo, y forma la parte no respirable que en cantidad de mas de setenta por ciento se encuentra en el aire atmosférico (s.v. azoe).

En la definición se reflejan las propiedades dadas al ázoe, pero se produce una confusión al equiparar 'fluido aeriforme' a gas ázoe, ya que es solo gas el sinónimo de fluido aeriforme, como se verá. La otra cursiva -método inusual en la definición- señala otro nuevo concepto, la materia del calor, o calórico. Por otro lado, la forma azote desaparece en el DRAE-1884, precisamente cuando se incorpora el término nitrógeno ${ }^{44}$.

Ahora retomemos la pareja fluido aeriforme - gas. El término gas había sido inventado por VAN HELMONT. Pero es LAVOISIER quien lo convierte en el nombre que designa el estado propio del aire, en "un nombre genérico que expresa el último grado de saturacion de qualquiera sustancia por el calóri-

42 Dice Gutiérrez Bueno (Morveau, Lavoisier, Berthollet y Fourcroy, 1788, 24): "Alguna vez, para evitar una significación siniestra, he añadido á la voz alguna cosa que la pudiese libertar de este peligro. Por esta razon, en vez de la palabra azote que se aplica al gas flogistico, digo azoote, que expresa la qualidad de ser no-vital, mucho mejor que azote, que en nuestro idioma significa cosa muy diversa". La forma azoote es la que aparece en A. CAPMANY (1805, s. v. azoth), pero aún no referida al nuevo concepto, sino definida como 'nombre que dan algunos alquimistas al azogue, ó mercurio'.

43 Como denominación del azogue, el término azoe aparece ya en AUTORIDADES (s. v. azogue), desde donde se remite a CovarRubIAS (1611), quien dice (s. v. açogue): 'Este nombre azogue es arábigo, de un verbo que vale correr; puede ser nombre griego, de $\xi \omega \eta$, zoe, vita et interposita G, zoge, y con el artículo arábigo, a-zogue'.

44 Sin embargo, a partir de ázoe se generan una serie de derivados, documentados en R. J. Domíncuez (1846) como son azótico, azótidos, azotífero, azotito, 
co" $^{\prime \prime}(1978,37)$. El adjetivo aeriforme había gozado hasta entonces del favor de los químicos, y se encuentra en expresiones como fluido aeriforme, substancia aeriforme, o estado aeriforme ${ }^{45}$. CAPMANY (1805) recoge gas como equivalente del francés gaz en su diccionario (s.v. gaz) ${ }^{46}$. En el DRAE-1817 aparecen por primera vez los dos términos rivales:

aeriforme. Quím. adj. que se aplica á las sustancias que se parecen al aire atmosférico siendo fluidos diferentes de él (s.v. aeriforme).

gas. s. m. El cuerpo que combinado con el calórico toma la forma del aire (s.v. gas).

La vacilación entre las dos voces sigue reflejándose en el ámbito lexicográfico, ya que aeriforme se define en el DRAE-1822 como 'Quím. adj. que se aplica á las sustancias cuando estan en forma de gas' (s.v. aeriforme), mientras que gas, en el DRAE-1852 remite a 'Flúido aeriforme'(s.v. aerifor$m e)^{47}$. Además, el adjetivo aeriforme rivaliza ya en algunos de los primeros textos con el también adjetivo gaseoso, aunque éste no se incorpora hasta el DRAE- $1837^{48}$.

El último de los términos al que me voy a referir, y que ya ha aparecido en las definiciones de fuego, ázoe y gas, es el de calórico. Nace, según LAvolSIER, para satisfacer la necesidad de diferenciar entre la causa y el efecto del calor, porque:

azotización, azotizado, azotizar, azotizarse, azotóides, azotoso, azotóxido, azoturo. En la traducción de LAVOISIER $(1798,55)$ se utiliza la forma azoético. En cuanto al término nitrógeno, también fue tenido en cuenta por LAVOISIER $(1798,39)$, junto al de alkalígeno, para designar la parte no respirable del aire, aunque acaba prefiriendo ázoe. En un primer momento, se utilizan términos como ayre viciado, ayre corrompido, o mofeta atmosférica; Vid. C. GARRICA (1995), donde estas y otras expresiones aparecen documentadas en los textos.

45 Así aparece en el título de una de las memorias de Martí I Franquès (1787), y es utilizado en diferentes ocasiones en las traducciones de MORVEAU, LAVOISIER, BerTHOLLET Y FOURCROY (1788) y de LAVOISIER (1798), compitiendo con el término gas.

46 Lo califica de voz de la química y le atribuye dos acepciones: 'La parte aromática volátil de una planta' y 'El ayre inflamable que sale de los cuerpos'.

47 Ya en el DRAE-1869 gas se define como 'Todo flúido aeriforme que permanece tal bajo presion y en la temperatura atmosférica ordinarias', mientras que aeriforme se define como 'Quím. Parecido al aire' a partir del DRAE-1884, y así se mantiene hasta la actualidad, aunque con marca de voz de la física.

48 Así ocurre en la traducción que GutiérRez Bueno haCe de Morveau, LaVoisier, BerThOllet Y Fourcroy $(1788,21)$. Por su parte, MarTí I Franquès (1787) utilizaba, además, las expresiones substancias aéreas (p. 12), fluido aereo (p. 17) y fluido elástico (p. 17). 
Siendo esta sustancia, sea la que fuese, la causa del calor, o en otros términos, siendo la sensacion que llamamos calor el efecto de la acumulacion de esta sustancia, no podremos en un lenguage riguroso darle el nombre de calor; porque una misma denominacion no puede expresar la causa y el efecto $(1798,3)$.

En un primer momento se utilizan las expresiones fluido ígneo y materia del calor ${ }^{49}$. El término calórico aparece por vez primera en el DRAE-1817:

Quím. El cuerpo simple ó materia elemental que produce la sensacion que se llama calor. Se tiene por un fluido extremamente sutil, que obedeciendo a las leyes de la atraccion, penetra ó abandona los poros de los cuerpos, dilatando ó contrayendo sus partes ó moléculas: se distingue en calórico combinado ó interpuesto segun el estado en que se le considera en los cuerpos (s.v. calórico).

Compárese la definición anterior con el ejemplo que LAVOISIER utiliza para explicar la naturaleza del calórico. Parece existir cierta influencia:

Si se meten en agua unos pedazos de madera de distintas especies, pero de igual volúmen, dicho fluido se introducirá poco á poco en sus poros, y se hincharán y aumentarán de peso; [...] en fin la porcion de agua embebida dependerá tambien de las moléculas constitutivas de la madera, y de su mayor ó menor afinidad con este líquido $(1798,13)$.

Y es que el calórico ha sido siempre un elemento difícil de comprender, como lo demuestra que la Academia se distancie en la definición del DRAE1832:

Quím. Segun la opinion comun de los químicos fluido sutilisimo que no se puede pesar ni reducir á espacio determinado, causa de la fluidez de los cuerpos y de la sensacion del calor (s.v. calórico).

Lo prueba también que a partir del DRAE-1884, ya con la marca de Fís. 'física' -desde el DRAE-1869-, se defina como 'Principio o agente hipotético [...]' -hasta la edición actual-, y que R. J. Domínguez (1846) y E. CHAO (1864) lo calificaran de 'fluido imponderable' (s.v. calórico) ${ }^{\text {s0 }}$. En cualquier caso, se

49 Pero estas denominaciones fueron desechadas, dice LAVOISIER (1798, 3-4), porque "alargan el discurso, le hacen mas lánguido, menos conciso, menos claro, y que por lo comun no representan ideas bastante exactas".

50 Según el DRAE-1992, fluido imponderable (s. v. fluido) es 'Fís. Cada uno de los agentes invisibles y de naturaleza desconocida que se han considerado como causa 
trata de una de las claves para explicar el fenómeno de la combustión en la química de Lavoisier, y por ello no podía estar ausente de la edición del DRAE1817.

\section{CONCLUSIÓN}

La penetración del lenguaje científico y técnico en los diccionarios es uno de los temas más controvertidos de la lexicografía. La tenue frontera entre lengua técnica y lengua común hace difícil el equilibrio entre la información lexicográfica y la enciclopédica. La Academia, durante todo el siglo XIX, fue acusada de parquedad en la incorporación de este tipo de términos.

Sin embargo, no se muestra tan remisa a la admisión de voces de la química. Es cierto que lo hace con retraso, pero también con decisión. El retraso puede ser achacado a las circunstancias históricas del primer decenio del siglo XIX; la decisión, a la precocidad con que las nuevas teorías químicas arraigaron en la Península, y al cuidadoso trabajo de los traductores, que adaptaron el léxico químico al españo| ${ }^{51}$. Una vez más, lengua y cultura, lengua y ciencia, deben aunarse para trazar una parte de la historia del léxico ${ }^{52}$.

Por otro lado, estos hechos refuerzan la importancia que la edición de 1817 tiene en la historia de la lexicografía académica, una edición prácticamente solo recordada por ser la primera que elimina el comentario de "reducido a un tomo", olvidando aspectos tan importantes como la reforma del sistema de marcas de uso o, como hemos visto, la introducción de determinadas voces de ciencias.

inmediata de los fenómenos eléctricos, magnéticos, luminosos y caloríficos, y se distinguían con el calificativo correspondiente'.

51 F. PetrecCA $(1992,252)$ menciona la química, junto a la botánica y la zoología, como casos de nuevas concepciones científicas que influyen con cierta rapidez en el diccionario, a pesar de la tardanza generalizada con que los movimientos culturales suelen reflejarse en las obras de referencia.

52 Esta es la línea que siguen mis trabajos sobre el léxico de la química (C. GarriGA, 1995, 1996b, 1997, 1997b y 1997c) y de la economía (C. GarRICA 1996), y los de F. Rodrícuez (1996 y 1997) sobre el léxico del ferrocarril o I. A. Moreno (1995 y 1995-96) sobre el de la electricidad. 
Bibliografía

Alvar, Manuel (1992): "El caminar del diccionario académico", Euralex'90, Barcelona, Vox-Biblograf, 3-27.

Alvar Ezquerra, Manuel (1987): "Presentación", en Esteban Terreros (1786), Diccionario castellano con las voces de Ciencias y Artes, Madrid, Viuda de Ibarra; facsímil en Madrid, Arco Libros, 5-16.

Alvar EzQuerra, Manuel (1992): "Tradición en los diccionarios del español", Revista Española de Lingüística, 22 / 1992, 1-23.

Álvarez de Miranda, Pedro (1992): "La Real Academia Española", Boletín de la Fundación Juan March, 225 / 1992, 3-16.

Anclada, Emilia y María Bargalló (1992): "Principios de lexicografía moderna en diccionarios del siglo XIX", en Actas del II Congreso Internacional de Historia de la Lengua Española, Madrid, Pabellón de España, 955-962. AUtORIDADES: ReAl ACADEMIA ESPAÑOLA (1726-1739): Diccionario de la lengua castellana, Madrid, Imprenta Real; facsímil en Madrid, Gredos, 1984.

BALDINGER, KURT (1985): "Lengua y cultura: su relación en la lingüística histórica", Revista Española de Lingǘística, 15, 2, 247-276.

BENSAUDE-VINCENT, BERNADETTE (1995): "Un regard européen sur la revolution chimique", en C. Puig-Pla, A. Camós, J. Arrizabalaga y Bernat, eds., Actes de les III trobades d'història de la ciència i de la tècnica als Països Catalans, Barcelona, SCHCT, 13-22.

Derthollet, Claude Louis (1795): Elementos del arte de teñir (trad. de Diego García Fernández), Madrid, Imprenta Real, [1791].

Calleja, Mạ. Carmen (1992): La farmacia en la llustración, Madrid, Akal.

CAPMANY, ANTONIO DE (1805): Nuevo diccionario francés español, Madrid, Imprenta de Sancha.

Clément, Jean Pierre (1993): Las instituciones científicas y la difusión de la ciencia durante la ilustración, Madrid, Akal.

ChaO, Eduardo (1864): Diccionario Enciclopédico de la Lengua Española, Madrid, Gaspar y Roig.

CovarRubias, Sebastián DE (1611): Tesoro de la lengua castellana o española, Madrid, Luis Sanchez; facsímil en Barcelona, Alta Fulla, 1987.

DomínGUeZ, Ramón Joaquín (1846): Diccionario nacional, Madrid, Miguel Guijarro.

Drae-1780: Real Academia Española: Diccionario de la lengua castellana, Madrid, J. Ibarra, $1^{\text {a }}$ ed.; facsímil en Madrid, Espasa-Calpe, 1991.

Drae-1783: ReAl Academia Española: Diccionario de la lengua castellana, Madrid, J. Ibarra, $2^{\mathrm{a}}$ ed.

Drae-1791: Real Academia Española: Diccionario de la lengua castellana, Madrid, Vda. de Ibarra, $3^{\underline{a}}$ ed. 
Drae-1803: Real Academia Española: Diccionario de la lengua castellana, Madrid, Vda. de Ibarra, 4므. ed.

Drae-1817: Real Academia Española: Diccionario de la lengua castellana, Madrid, Imprenta Real, 5a ed.

Drae-1822: ReAL ACADEMIA ESPAÑOLA,:Diccionario de la lengua castellana, Madrid, Imprenta Nacional, $6^{\underline{a}}$ ed.

Drae-1837: ReAl ACADEMIA Española: Diccionario de la lengua castellana, Madrid, Imprenta Nacional, 8aㅡ ed.

Drae-1852: ReAl ACADEMIA Española: Diccionario de la lengua castellana, Madrid, Imprenta Nacional, $10^{\underline{a}}$ ed.

Drae-1869: Real Academia Española: Diccionario de la lengua castellana, Madrid, M. Rivadeneyra, $11^{\underline{a}}$ ed.

Drae-1884: Real Academia Española: Diccionario de la lengua castellana, Madrid, Gregorio Hernando, $12^{\underline{a}}$ ed.

Drae-1992: Real ACAdemia Española: Diccionario de la lengua española, Madrid, Espasa-Calpe, $21^{\stackrel{a}{a}}$ ed.

Esqué, Ma . Dolors y ANNA Má. CARMONA (1995): "Aportació dels investigadors de la història de la ciència als Països Catalans al coneixement de la figura d'Antoni Martí i Franquès", en C. Puig-Pla, A. Camós, J. Arrizabalaga, Bernat, eds., Actes de les III trobades d'història de la ciència i de la tècnica als Països Catalans, Barcelona, SCHCT, 391-393.

Esteva de SAGReRA, JUAN (1991): La química sagrada. De la alquimia a la química en el siglo XVII, Madrid, Akal.

Fries, Dagmar (1989): La Real Academia Española ante el uso de la lengua, Madrid, SGEL.

GaGo, Ramón (1982): "Introducción", en L. A. Lavoisier, Tratado elemental de química, Madrid, Alfaguara, XIII-LV.

Gago, Ramón (1990): "Luis Proust y la cátedra de química de la Academia de Artillería de Segovia", en L. Proust, Anales del Real Laboratorio de química de Segovia, Segovia, A. Espinosa, 1791; en el facsímil de Segovia, Academia de Artillería, 3-51.

Gago, Ramón; José luis Carrillo y luis García Ballester, (1974): "Juan Manuel de Aréjula y la nueva nomenclatura química", Cuadernos de Historia de la Medicina Española, 13, 273-295.

Garriga, Cecilio (1995): "Apuntes sobre la incorporación del léxico de la química al español: la influencia de Lavoisier", en 1648-1815: L' "universalité» du français et sa présence dans la Péninsule Ibérique, Tarragona, en prensa.

Garrica, Cecilio (1996): "Notas al léxico económico del siglo XVIII", en A. Alonso, L. Castro, B. Gutiérrez y J. A. Pascual, eds., Actas del III Congreso Internacional de Historia de la Lengua Española, Madrid, Arco-Libros, 1279-1288. 
Garrica, Cecilio (1996b): "Luis Proust y la consolidadción de la terminología química en español", Actas del VI Congreso de la Sociedad Española de Historia de la Ciencia y de las Técnicas, Segovia, en prensa.

Garriga, Cecilio (1997): "El «Diccionario Universal de Física» de Brisson (1796-1802) y la fijación lexicográfica de la terminología química en español", en Actas del IV Congreso Internacional de Historia de la lengua española, en prensa.

Garrica, CeCilio (1997b): "La recepción de la nueva nomenclatura química en español", Grenzgäenge, en prensa.

GarRICA, CeCilio (1997c): "Química, enseñanza y divulgación de la terminología: los Elementos / Lecciones de química teórica y práctica de Morreau, Maret y Durande", en La Història dels llenguatges iberoromànics d'especialitat (segles XVII-XIX), Barcelona, Universitat Pompeu Fabra, en prensa.

GoupIL, MiCHeLle, ed., (1992): Lavoisier et la révolution chimique, Sabix, École polytechnique.

Gutiérrez Cuadrado, Juan y José luis Peset (en prensa): Metro y kilo: el sistema métrico decimal en España, Madrid, Akal.

Herrero, Ma Dolores (1993): Cañones y probetas en el Alcázar. Un siglo de historia del Real Colegio de Artillería de Segovia, Segovia, Patronato del Alcázar.

Laín Entralco, Pedro (1978): Historia de la medicina, Salvat, Barcelona.

LaVoIsier, ANTOINe LaURent (1788): "Memoria. Sobre la necesidad de perfeccionar y reformar la nomenclatura de la química, leida en la junta pública de la Academia Real de las Ciencias de Paris, el 18 de abril de 1787", en Morveau, Lavoisier, Berthollet y Fourcroy, Método de la nueva nomenclatura química (trad. Pedro Gutiérrez Bueno), Madrid, Antonio de Sancha, [1787].

Lavolsier, ANTOINe LaURent (1798): Tratado elemental de química (trad. de Juan Manuel Munárriz), Madrid, Imprenta Real, [1789].

Lázaro Carreter, Fernando (1980): "El primer diccionario de la Academia", en Estudios de lingüística, Barcelona, Crítica, 83-148.

LÁZARO CARRETER, FERNANDO (1985): "Neologismo y purismo", en Las ideas lingüísticas en España durante el siglo XVIII, Barcelona, Crítica, 245-282.

López PIÑERO, José Ma . (1979): Ciencia y técnica en la sociedad española de los siglos XVI Y XVII, Barcelona, Labor.

LóPEz PIÑERO, JOSÉ Mã . (1982): La ciencia en la historia hispánica, Madrid, Salvat.

LóPez PIÑERO, José Ma (1992): "Introducción", en La ciencia en la España del s. XIX, Madrid, Marcial Pons, 11-18. 
MARTí I Franquès, ANTONI (1787): "Sobre algunas producciones que resultan de la combinación de varias sustancias aeriformes", en Antoni Quintana (1935), Antoni de Martí i Franquès; memòries originals, estudi biogràfic $i$ documental, Barcelona, Acadèmia de Ciències i Arts, 11-20.

MARTi I FRANQUÈs, ANTONI (1790): "Sobre la cantidad de aire vital que se halla en el aire atmosférico y sobre varios métodos de conocerla", en Antoni Quintana (1935), Antoni de Martí i Franquès; memòries originals, estudi biogràfic i documental, Barcelona, Acadèmia de Ciències i Arts, 21-36.

MoRENO, José ANTONIO (1995): "J. A. Nollet: un nuevo léxico para una nueva ciencia", en 1648-1815: L' "universalité» du français et sa présence dans la Péninsule Ibérique, Tarragona, en prensa.

MORENO, José ANTONIO (1995-96): "La recepción del léxico de la electricidad en el DRAE: de Autoridades a 1884", Revista de lexicografía, II, 73-97.

Morveau, L.; A. L. Lavoisier, Cl. Berthollet, A. F. Fourcroy (1788): Método de la nueva nomenclatura química (trad. Pedro Gutiérrez Bueno), Madrid, Antonio de Sancha, [1787].

Nieto, Acustí (1996): "Martí i Franquès, Carbonell i Bravo, i els usos de la nova química a la Catalunya II-lustrada", en Mercè Izquierdo y otros, eds., Lavoisier $i$ els orígens de la química moderna, 200 anys després (1794-1994), Barcelona, SCHCT, 159-184.

PAsCual, José ANTONIO y JuAn GutiérRez Cuadrado (1992): "Prólogo a propósito de las actas del Congreso Literario Hispano-Americano de 1892", en Congreso Literario Hispano-Americano (1892), Madrid, Instituto Cervantes - Pabellón de España - Biblioteca Nacional, IX-XXXI.

Pellón, Inés y Ramón GaGo (1994): Historia de las Cátedras de Química y Mineralogía de Bergara a finales del siglo XVIII, Bergara, Ayuntamiento.

Peset, Mariano y José LuIS Peset (1992): "Las universidades españolas del s. XIX y las ciencias", en José M ${ }^{a}$. López Piñero, ed., La ciencia en la España del s. XIX, Madrid, Marcial Pons, 19-49.

Petrecca, Francisco (1992): "Taxonomía científica y discurso lexicográfico", BRAE, LXII, 251-267.

PORTELA, EUGENIO y AMPARO SOler (1992): La química española en el s. XIX, en José Ma . López Piñero, ed., La ciencia en la España del s. XIX, Madrid, Marcial Pons, 85-107.

Proust, LuIs (1795): Anales del Real Laboratorio de Química de Segovia (tomo II), Segovia, A. Espinosa; ed. facsímil de Ramón Gago, Segovia, Academia de Artillería, 1990.

QUINTANA, ANTONI (1935): Antoni de Martí i Franquès; memòries originals, estudi biogràfic i documental, Barcelona, Acadèmia de Ciències i Arts.

Riera, Santiaco (1992): Tecnología en la llustración, Madrid, Akal. 
Rodrícuez, Francisco (1996): "El léxico de los «caminos de hierro» en español", en A. Alonso, L. Castro, B. Gutiérrez y J. A. Pascual, eds., Actas del III Congreso Internacional de Historia de la Lengua Española, Madrid, Arco-Libros, 1511-1519.

Rodrícuez, Francisco (1997): Introducción y desarrollo del léxico del ferrocarril en la lengua española, Barcelona, Universidad.

Rolc, CaRmen (1995): "El Nuevo diccionario francés-español de Antonio de Capmany", en Francisco Lafarga y otros, eds., La traducción. Metodología-historia-literatura. Ámbito hispanofrancés. Barcelona, PPU, 75-80.

Seco, Manuel (1987): "El nacimiento de la lexicografía moderna no académica", en Estudios de lexicografía española, Madrid, Paraninfo, 129-151.

Seco, Manuel (1987b): "Ramón Joaquín Domínguez", en Estudios de lexicografía española, Madrid, Paraninfo, 152-164.

Seco, Manuel (1991): "Introducción", en Real Academia Española (1780), Diccionario de la lengua castellana, Madrid, J. Ibarra; facsímil en Madrid, Espasa-Calpe, 1991, III-XII.

SimÓN, MARTA; Mercè IzQUierdo (1995): "Lavoisier i el llenguatge simbòlic", en C. Puig-Pla, A. Camós, J. Arrizabalaga, Bernat, eds., Actes de les III trobades d'història de la ciència i de la tècnica als Països Catalans, Barcelona, SCHCT, 377-384.

Terreros, Esteban (1786): Diccionario castellano con las voces de Ciencias y Artes, Madrid, Viuda de Ibarra; facsímil en Madrid, Arco Libros, 1987.

VIÑAZA, CONDE DE LA (1893): Biblioteca histórica de la Filología Castellana, Madrid, Manuel Tello; facsímil en Madrid, Atlas, 1978.

WojtKowiAk, B. (1987): Historia de la química, Zaragoza, Acribia. 